\title{
Reliability-Based Decision Fusion in Multimodal Biometric Verification Systems
}

\author{
Krzysztof Kryszczuk, Jonas Richiardi, Plamen Prodanov, and Andrzej Drygajlo
}

Signal Processing Institute, Swiss Federal Institute of Technology, 1015 Lausanne, Switzerland

Received 18 May 2006; Revised 1 February 2007; Accepted 31 March 2007

Recommended by Hugo Van Hamme

We present a methodology of reliability estimation in the multimodal biometric verification scenario. Reliability estimation has shown to be an efficient and accurate way of predicting and correcting erroneous classification decisions in both unimodal (speech, face, online signature) and multimodal (speech and face) systems. While the initial research results indicate the high potential of the proposed methodology, the performance of the reliability estimation in a multimodal setting has not been sufficiently studied or evaluated. In this paper, we demonstrate the advantages of using the unimodal reliability information in order to perform an efficient biometric fusion of two modalities. We further show the presented method to be superior to state-of-the-art multimodal decision-level fusion schemes. The experimental evaluation presented in this paper is based on the popular benchmarking bimodal BANCA database.

Copyright (c) 2007 Krzysztof Kryszczuk et al. This is an open access article distributed under the Creative Commons Attribution License, which permits unrestricted use, distribution, and reproduction in any medium, provided the original work is properly cited.

\section{INTRODUCTION}

Biometric verification systems deployed in a real-world environment often have to contend with adverse conditions of biometric signal acquisition, which can be very different from the carefully controlled enrollment conditions. Examples of such conditions include additive acoustic noise that may contaminate the speech signal, or nonuniform directional illumination that can alter the appearance of a face in a two-dimensional image. Methods of signal conditioning and normalization as well as tailor-made feature extraction schemes help reduce the recognition errors due to the degraded signal quality, however they invariably do not eliminate the problem (see, e.g., $[1,2]$ ). Combining independent biometric modalities has proved to be an effective manner of improving accuracy in biometric verification systems [3]. A fusion of discriminative powers of independent biometric traits, not equally affected by the same environmental conditions, affords robustness to possible degradations of acquired biometric signals.

Common methods of classifier fusion at the decision level employ a prediction of the average error of each of the unimodal classifiers, typically based on resampling of the training data $[3,4]$. This average modality error information can be applied to weight the unimodal classifier decisions during the fusion process. The drawback of this approach is that it does not take into account the fact that individual decisions depend on the acquisition conditions of the data presented to the expert as well as on the discriminating skills of the classifier. In the case of two available modalities, this approach is also equivalent to the systematic use of the decisions of the more accurate modality and thus defies the purpose of fusion.

Signal quality and impostor/client score distributions have been used to train weights for classifier combination in multimodal biometric verification in [5]. The quality measures were used during the training of the decision module. However, the quality measures for particular modalities were subjective quality tags manually assigned to the training and testing data. Also, the causal relationships between the environmental conditions and the classification results were not deliberately modeled.

In this paper, we investigate an alternative approach to dynamic decision weighting in multimodal biometric fusion. We propose to compare the single decision reliability estimates in order to maximize the probability of making a correct fusion decision. The measure of reliability is defined in probabilistic terms and expresses the degree of trust one can have in a particular unimodal classifier decision. We proposed a method of modeling influence of signal quality on 
classifier scores and decisions with application to classifier error prediction in [6]. The method uses a Bayesian network trained to predict classification errors given the classification score, classifier decision, and automatically obtained auxiliary information about the quality of the biometric data presented to the unimodal classifier. A system using a speech expert (a speech classifier combined with a decision reliability estimator) was shown to significantly reduce the total classification error rate for speech-based biometric verification in a sequential repair strategy. In the presence of a second biometric trait available, a sequential repair strategy can be replaced by a parallel one where the unreliable decision of one unimodal classifier can be replaced by a more reliable decision for another modality. In [7], we presented an embodiment of this parallel multimodal repair strategy, using speech and face experts and a multimodal fusion module. The proposed method yielded higher accuracy than any unimodal system alone through prediction and correction of the verification decisions. The results reported in this work were a proof of concept, demonstrated on an artificially created chimerical database that by default contained as many classifier errors as correct decisions. This is obviously not the case in real applications where by definition the number of errors is minimized. In this paper, we present the application of the proposed method to a real multimodal database (BANCA), where both modalities come from the same individual. In [8], Poh and Bengio presented a method of estimating the confidence of single classifier decisions using the concept of margins, which proved to grant good fusion performance in a multimodal scenario. In the current paper, we show that our method of reliability based fusion outperforms the margin approach, thanks to the use of quality measures and the modeling of their relationship with classifier decisions.

This paper is structured as follows: in Section 2, we summarize the theoretical framework of reliability estimation using Bayesian networks and signal-level quality measurements. In Section 3 we discuss details of the multimodal database and experimental protocols. Sections 4 and 5 detail the speaker and face verification systems together with corresponding algorithms to estimate signal quality. Section 6 introduces the decision-level scheme for multimodal fusion with reliability estimates. Section 7 presents the experimental results and their discussion, and finally Section 8 concludes the paper.

\section{VERIFICATION DECISION RELIABILITY ESTIMATION}

\subsection{Bayesian networks for reliability modeling}

We define decision reliability for a given modality MR as the probability that the classifier for this modality has taken a correct verification decision given the available evidence, that is, the probability $\mathrm{P}(\mathrm{MR} \mid \mathrm{E})$. The evidence $\mathrm{E}$ that provides information about the state of MR can come from several sources: signal domain, feature domain, score domain, or decision domain itself. In the present work, for each modality we use a vector of signal-domain quality measures QM, classifier score information Sc, and classified identity CID (CID $=1$ if the score for this biometric presentation is above

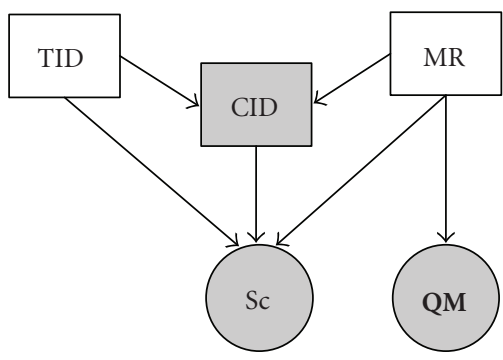

FIGURE 1: Bayesian network for modality decision reliability estimation.

the decision threshold, otherwise CID $=0$ ). Furthermore, in training a decision reliability estimator, it is crucial to provide the ground truth about the user true user identity TID (TID $=1$ if the biometric presentation really belongs to the claimed client, otherwise TID =0) so that the influence of the event "the user is a client" on other variables can be taken into account in modeling. Thus, $\mathrm{MR}=1$ represents "the decision from this modality is reliable" (i.e., TID = CID) and $\mathrm{MR}=0$ represents the opposite statement. These sources of information and their interrelations are modeled probabilistically using the Bayesian network shown on Figure 1. In this model, the true user identity (TID) influences the classified user identity (CID), and the decision reliability for this modality (MR) also impacts the classifier's decision (CID). $\mathrm{MR}, \mathrm{CID}$, and TID are all interdependent with the classifier score Sc, and MR is related to the observed quality measures QM. It should be noted that the number of nodes could be reduced by removing the TID node, since functionally the state of the CID and MR binary variables is sufficient to recover TID. For more details on the rationale behind the creation of this model, originally used in speaker verification, the reader is referred to [6]. This model differs from the generative approach in [9] and the normalization approach in [10], as we take into account the distribution of scores for correct and erroneous base classifier decisions, and not only for client and impostors. More importantly, we use a measure of signal quality.

The Bayesian network is used for providing values for $\mathrm{P}(\mathrm{MR} \mid \mathrm{E})$, which in our case is $\mathrm{P}$ (MR | CID, Sc, QM). This marginal probability, which we call the decision reliability, expresses the probability that the classifier for this modality has taken a correct/wrong decision given available evidence. Inference on $\mathrm{P}(\mathrm{MR} \mid \mathrm{CID}, \mathrm{Sc}, \mathbf{Q M})$ is only possible once the conditional distribution parameters for the variables have been learned from training examples. The network parameters can be estimated using a maximum likelihood (ML) training technique [11]. Figure 2 provides a diagram of a modality expert consisting of the baseline classifier for a modality and the corresponding Bayesian network estimating the decision reliability. The classifier part of the expert is trained from held-out data which is not used again (see Section 7). The reliability estimator is trained on sets of variable values (CID, Sc, QM, TID) obtained by feeding biometric data in diverse environmental conditions to the classifier 


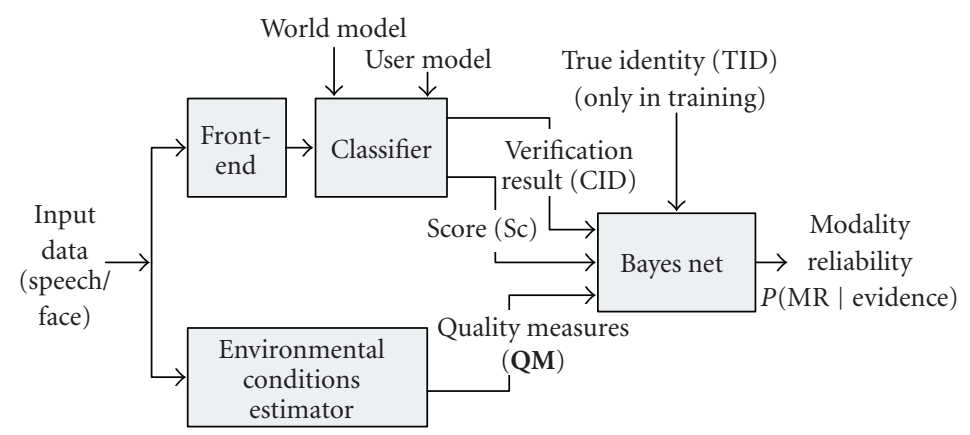

FIgURE 2: Modality expert with modality classifier and modality reliability estimator.

and the environmental conditions estimator. The environmental conditions estimator provides values for the QM variable as described in Sections 4 and 5.

It should be noted that TID is only observed during training.

The probabilistic decision reliability for each modality, for example, for speech $\mathrm{P}\left(\mathrm{MR}_{s}=1 \mid \mathrm{CID}, \mathrm{Sc}, \mathrm{QM}\right)$ and for face $\mathrm{P}\left(\mathrm{MR}_{f}=1 \mid \mathrm{CID}, \mathrm{Sc}, \mathbf{Q M}\right)$ can be used to enhance the accuracy of the final of the multimodal verification system.

\subsection{Modeling confidence with margins}

In the process of reliability estimation we seek a measure of how likely it is that the classifier took the correct decision. Many confidence measures have been proposed for speaker verification [12]; for example, the computation of a margin provides such a confidence measure [8]. It is an intuitive and appealing way of estimating the reliability of a decision for any biometric modality. For given classifier score Sc the margin function is defined as

$$
M(\mathrm{Sc})=|\mathrm{CR}(\mathrm{Sc})-\mathrm{CA}(\mathrm{Sc})|
$$

where $\mathrm{CR}(\mathrm{Sc})$ and $\mathrm{CA}(\mathrm{Sc})$ are, respectively, the identity claim rejection and acceptance accuracies at a given threshold (score). The absolute value of the difference in observed probabilities represents a frequentist estimate of the certainty of the classifier in having chosen one decision over the alternative one. In the general case, the function $M(\mathrm{Sc})$ is estimated empirically on a dataset not used during the training and testing phases. In our case, the margin function was estimated on the development dataset. It must be noted that the frequentist approach to reliability estimation is valid only under the assumption that the scores of the testing data originate from similar distribution as the scores originating from the development set. In our experiments that assumption is supported by the similarities in the structure of the development and testing datasets.

\section{DATABASE AND EXPERIMENTAL CONDITIONS}

We used face images and speech data from the BANCA database, English part, which has recently become a bench- marking multimodal database. BANCA contains data collected from a pool of 52 individuals, 26 males and 26 females. In this paper, we adhere to the evaluation protocol P. For the details on the BANCA database and the associated evaluation protocol the reader is referred to [13].

\subsection{Face modality data}

The face data from the BANCA database consists of images collected in three different recording conditions: controlled, degraded, and adverse. For each of the recording condition, four independent recording sessions were organized, making a total of 12 sessions. The faces in the images were localized manually, cropped out and normalized geometrically (aligned eye positions) and photometrically (histogram normalization). Examples of thus prepared images of controlled, degraded, and adverse quality are presented in Figure 3.

\subsection{Speech modality data}

The BANCA database provides a large amount of training data per user: 2 files per session (about 20 seconds. each) $\times 2$ microphones $\times 12$ sessions. In our case, we used only the data from microphone 1 . The first 4 sessions are in "clean" conditions, the next 4 sessions are in "degraded" conditions, and the last 4 sessions are in "adverse" conditions. The only preprocessing we perform before feature extraction is speech/pause detection based on energy.

\subsection{Bimodal protocol}

While being a bimodal database, BANCA has no predefined reference protocols for multimodal testing. However, predefined protocols are provided for single modality testing scenarios. In our experiments we make use of the $\mathrm{P}$ protocol for unimodal testing since it closely corresponds to our assumptions about the experimental design. Namely, it involves training the classification models using high-quality data recorded in the controlled conditions, and testing using data acquired in the controlled as well as deteriorated conditions. The details of the testing protocol $\mathrm{P}$ can be inspected in [13]. The protocol declares that all database data 


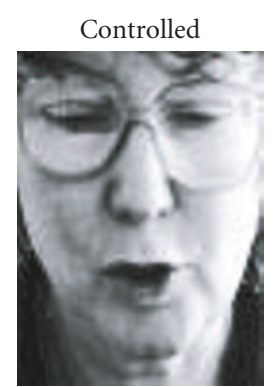

(a)

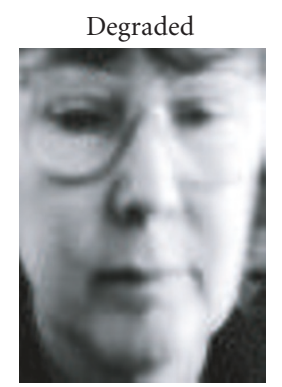

(b)

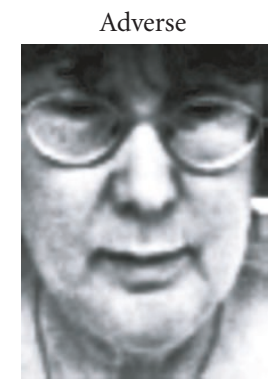

(c)

FIGURE 3: Example of the images collected in the controlled, degraded, and adverse scenarios (left to right) from the same individual.

have to be subdivided into two subsets, g1 and g2, consisting of different users. While data from one dataset is used for user model training and testing, the other dataset (a development set) may be used for parameter tuning. In accord with this directive, we use the development set to adjust the decision thresholds for the test set, but also to train the Bayesian networks used in the reliability estimation routines. The unimodal protocol strictly defines the assignment of user data to the genuine access or impostor access pools. We respect this assignment and in order to do so reduce the amount of client face images to one per access (as opposed to the available five) in order to match the amount of speech data at hand. In this way, we maintain the compatibility with the $\mathrm{P}$ protocol and at the same time we overcome the problems related to the use of the chimerical databases [8].

\section{SPEAKER VERIFICATION AND QUALITY MEASURES}

The speech-based classifier is trained by using training files from session 1 as defined by the BANCA P protocol. 12 melfrequency cepstral coefficients with first- and second-order time derivatives are extracted with cepstral mean normalization. Using the ALIZE toolkit [14], a world Gaussian mixture model (GMM) of 200 Gaussian components with diagonal covariance matrices is trained from the pooled training features of all users. The user models are then MAP-adapted from the world model using the user-specific training data from session 1 . When training and testing on g1, the thresholds are estimated on g2 a posteriori (corresponding to the equal error rate (EER) point), then used on g1, and viceversa for g2. This classifier provides the CID and Sc variables to the reliability estimator, and its performance is consistent with baseline GMM results available in the litterature on the BANCA P protocol.

The signal-to-noise ratio (SNR) contains information about the level of acoustic noise in the speech signal, which is one of the main factors of signal quality degradation. Thus, the quality measure used for speech is an SNR-related measure. The SNR is defined as the ratio of the average energy of the speech signal divided by the average energy of the acoustic noise in $\mathrm{dB}$. We perform speech/pause segmentation using an algorithm based on the "Murphy algorithm" described in [15]. We then assume that the average energy of pauses is as- sociated with that of noise. Our SNR-related quality measure (SQM) is given by the formula

$$
\mathrm{SQM}=10 \log _{10} \frac{\sum_{i=1}^{N} I s(i) s^{2}(i)}{\sum_{i=1}^{N} \operatorname{In}(i) s^{2}(i)},
$$

where $\{s(i)\}, i=1, \ldots, N$ is the acquired speech signal containing $N$ samples, $I s(i)$ and $\operatorname{In}(i)$ are the indicator functions of the current sample $s(i)$ being speech or noise during pauses (e.g., $I s(i)=1$ if $s(i)$ is a speech sample, $I s(i)=0$ otherwise). Other experiments with a speech quality measure using entropy-based speech/pause segmentation are described in [12].

\section{FACE VERIFICATION AND QUALITY MEASURES}

In our experiments we have used a face verification scheme implemented in a similar fashion as presented in [16] with the decision threshold set to training EER. The images from the BANCA database (English part) were used to build the world model (520 images, $26+10$ individuals (g1 or g2 subsets, resp.), 384 Gaussians in the mixture). Client models were built using world model adaptation [15]. The images used in the experiments were cropped, photometrically normalized by histogram equalization, and scaled to the size of $64 \times 80$ pixels. The average half-total error rate (HTER) [8] of the used classifier is comparable to the state-of-the-art algorithms [17].

\subsection{Correlation with an average face image}

The goal of the relative quality measurement is to determine to what degree the quality of the testing image departs from that of the training images. The quality of the training images can be modeled by creating an average face template out of all the face images whose quality is considered as reference. We have built an average face template using PCA reconstruction, in similar fashion as described in [16]. Specifically, we have used the first eight averaged Eigenfaces to build the template. Two average face templates built of images from the BANCA database are shown in Figure 4.

For the experiments presented in this paper, we have created two average face templates from the training images prescribed by the P protocol (clients from the groups g1 and 


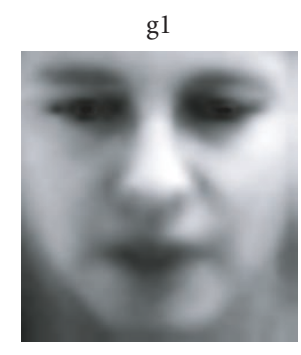

(a)

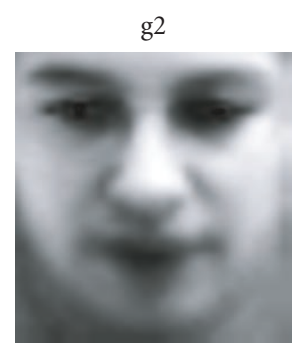

(b)
FIGURE 4: Average face template built using training images defined in the BANCA P protocol for the datasets $\mathrm{g} 1$ and g2, respectively.

g2). It is noteworthy that the average face templates created from the images of two disjoint sets of individuals are strikingly similar. It is also apparent that high-resolution details are lost, while low-frequency features, such as head pose and illumination, are preserved. Therefore, in order to obtain a measure of similarity of low-frequency face images, we propose to calculate the Pearson's cross-correlation coefficient between the face image I whose quality is under assessment, and the respective average face template AVF:

$$
\mathrm{FQM}_{1}=\rho(\mathrm{AVF}, I)
$$

\subsection{Image sharpness estimation}

The cross-correlation with an average image gives an estimate of the quality deterioration in the low-frequency features. At the same time that measure ignores any quality deterioration in the upper range of spatial frequencies. The absence of high-frequency image details can be described as the loss of image sharpness. In the case of the BANCA database, the images collected in the degraded conditions suffer from a significant loss of sharpness. An example of this deterioration can be found in Figure 3. In order to estimate the sharpness of an image I of $x \times y$ pixels, we compute the mean of intensity differences between adjacent pixels, taken in both the vertical and horizontal directions:

$$
\begin{aligned}
\mathrm{FQM}_{2}=\frac{1}{2}( & \frac{1}{(x-1) y} \sum_{m=1}^{y} \sum_{n=1}^{x-1}\left|p_{n, m}-p_{n+1, m}\right| \\
& \left.+\frac{1}{(y-1) x} \sum_{m=1}^{y-1} \sum_{n=1}^{x}\left|p_{n, m}-p_{n, m+1}\right|\right) .
\end{aligned}
$$

\section{MULTIMODAL DECISION FUSION WITH RELIABILITY INFORMATION}

Figure 5 presents the schematic diagram of the system used in our experiment. Biometric data of an individual (face image and speech) are corrupted by extraneous conditions: in the case of speech additive noise, and in the case of the face departure from the nominal illumination and image sharpness. The speech and face acquisition process consists of all the signal-domain preprocessing and normalization steps $[6,18]$ that make the speech data and face image usable for

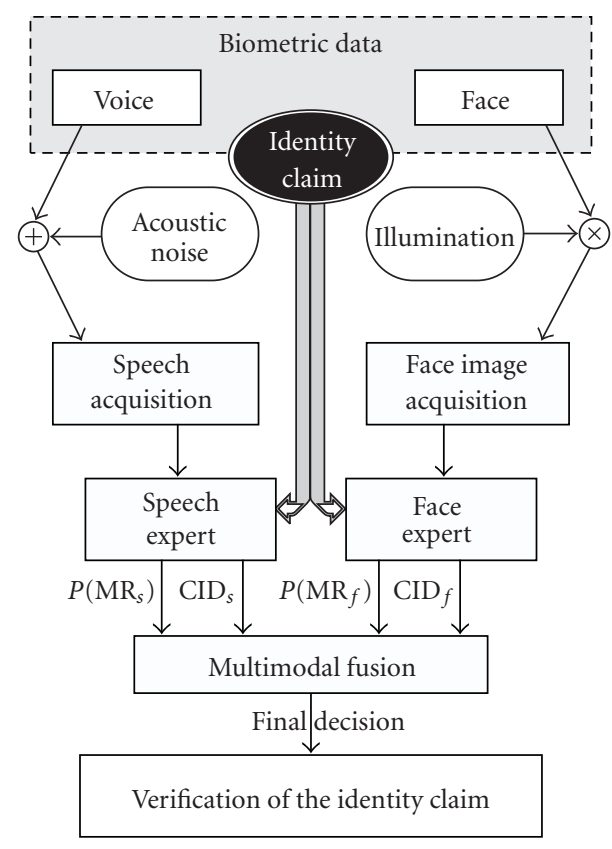

FIGURE 5: Multimodal biometric verification system with reliability information.

TABLE 1: Decision table for multimodal decision module.

\begin{tabular}{l|cl}
\hline Face & Speech & Final decision \\
\hline $\mathrm{CID}_{f}=1$ & $\mathrm{CID}_{s}=1$ & 1 \\
$\mathrm{CID}_{f}=1$ & $\mathrm{CID}_{s}=0$ & $\begin{array}{l}1: \text { if } \mathrm{P}\left(\mathrm{MR}_{f}=1\right)>\mathrm{P}\left(\mathrm{MR}_{s}=1\right), \\
\end{array}$ \\
$\mathrm{CID}_{f}=0$ & $\mathrm{CID}_{s}=1$ & $\begin{array}{l}1: \text { if } \mathrm{P}\left(\mathrm{MR}_{f}=1\right)<\mathrm{P}\left(\mathrm{MR}_{s}=1\right), \\
0\end{array}$ \\
$\mathrm{CID}_{f}=0$ & $\mathrm{CID}_{s}=0$ & 0
\end{tabular}

the modality experts (see Figure 2). Each of the experts accepts two inputs: the conditioned data from the acquisition process and the identity claim. On the output, the experts produce verification decisions $\mathrm{CID}_{f}$ and $\mathrm{CID}_{s}$ (for face and speech, resp.) and modality reliability information $\mathrm{MR}_{f}$ and $\mathrm{MR}_{s}$, on the base of which the multimodal decision module (see Table 1) returns the final verification decision.

The fusion of the verification information coming from face and speech experts is performed using the classifier decisions and the modality reliability data. If both experts agree on the decision, the decision is preserved. If they are in disagreement, the decision is taken in accordance to Table 1. This decision selection scheme is designed to maximize the probability of making a correct decision.

\section{EXPERIMENTAL RESULTS}

We tested the performance of the unimodal experts and the reliability they produce, as well as the use of the reliability information in the multimodal decision-level fusion process. 
TABle 2: Decision reliability classification accuracy. All results are in percent.

\begin{tabular}{l|rrrrr}
\hline Modality & $\mathrm{acc}_{\mathrm{CA}}$ & $\mathrm{acc}_{\mathrm{CR}}$ & $\mathrm{acc}_{\mathrm{FA}}$ & $\mathrm{acc}_{\mathrm{FR}}$ & $\mathrm{acc}_{\mu}$ \\
\hline Speech $_{\text {rel }}$ & 79.4 & 72.9 & 94.4 & 86.1 & 83.2 \\
Speech $_{\text {margin }}$ & 51.7 & 55.1 & 100.0 & 97.2 & 76.0 \\
Face $_{\text {rel }}$ & 54.7 & 54.5 & 75.6 & 92.7 & 69.4 \\
Face $_{\text {margin }}$ & 48.2 & 67.8 & 75.9 & 78.5 & 67.6 \\
\hline
\end{tabular}

\subsection{Unimodal reliability on speech and face data}

The baseline classifiers were trained and tested on g1 according to protocol P. The test results on g1 were used as training data for the reliability model. Then, the baseline classifiers were trained and tested on $\mathrm{g} 2$ according to protocol $\mathrm{P}$, and the test results on g2 were used as test data for the reliability models. This procedure is repeated, inverting g1 and g2, and the accuracies are computed as the mean of the errors for $\mathrm{g} 1$ and $\mathrm{g} 2$.

We use the classical definition of accuracy as

$$
\operatorname{acc}_{x}=\frac{n \text { Correct Classifications }(x)}{n \text { Samples }(x)}
$$

where $x$ stands for correct accept (CA), correct reject (CR), false accept (FA), or false reject (FR). Since the number of cases of CA, CR, FA, FR is unbalanced in the training and testing set, we also define a mean accuracy over all 4 cases as

$$
\operatorname{acc}_{\mu}=\frac{1}{4}\left(\operatorname{acc}_{\mathrm{CA}}+\operatorname{acc}_{\mathrm{CR}}+\operatorname{acc}_{\mathrm{FA}}+\mathrm{acc}_{\mathrm{FR}}\right)
$$

so that the reliability measure will be penalized if it performs well only in certain cases.

As the accuracies in Table 2 show, there is a large discrepancy between the classification accuracy for correct decisions and false decisions, in favor of false decisions. This tendency is persistent over both modalities and both datasets (g1 and g2). Taking into consideration the fact that the use of a real database (BANCA) is bound to produce far more correct than erroneous decisions, the unimodal decision rectification scheme as described in [7] could not be applied.

Figure 6(a) shows the relationship between the decision reliability (reliability threshold) for each modality and the corresponding error rates for the observations whose reliability is equal or greater than the reliability threshold, in terms of 1-HTER. The monotonous increase of (1-HTER) as a function of the reliability threshold shows that indeed a higher reliability estimate positively correlates with the chances of making a correct classification decision. In Figure 6(b) we show the relative count of decisions whose reliability is equal to or greater than the given reliability value, as a function of the reliability threshold. Table 3 gives the average reliability of both modalities. As the graphs and tabulated means show, in our experiments the speech modality was on average more reliable than the face modality.

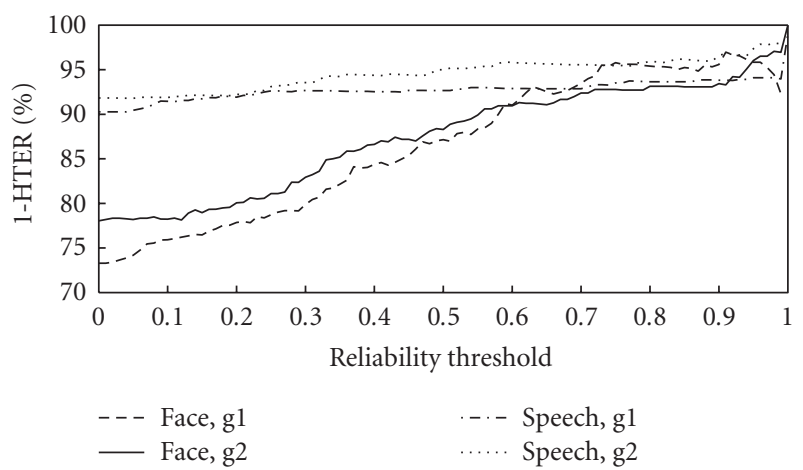

(a)

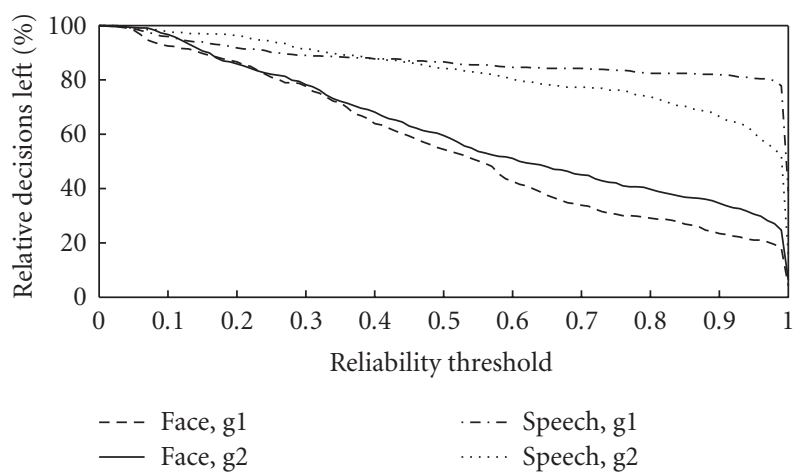

(b)

FIGURE 6: Distribution of reliability values on the g1 and g2 datasets for speech and face.

TABLE 3: Mean reliability estimates for face and speech (in percent).

\begin{tabular}{l|ccc}
\hline Modality & g1 & g2 & avg. \\
\hline Speech & 76.4 & 69.6 & 73.0 \\
Face & 51.5 & 54.1 & 52.8 \\
\hline
\end{tabular}

\subsection{Multimodal experiments}

Since the work presented in this paper focuses on decisionlevel fusion, all fusion experiments make use of only unimodal decisions obtained from the classifiers described in Sections 4 and 5. In order to preserve compatibility with the BANCA protocol, we report the fusion results in terms of HTER separately for each of the datasets g1 and g2, as well as the averaged results (g1 and g2). The theoretical limit of the accuracy improvement achieved by multimodal fusion can be expressed by computing the oracle accuracy, that is, assuming that the correct decisions and errors of each of the unimodal classifiers are labeled. The oracle scenario therefore yields false decisions only if both of the unimodal classifiers were wrong. Oracle results are an efficient way of telling the classifier errors due to data modeling imperfections from errors due to the inherent data problems (e.g., nondiscriminative features). This interpretation, however, is straightforward only if both classifiers operate on 
TABLE 4: Error rates (HTER, FAR (false accept rate), FRR (false reject rate)), in percent, for speech and face baseline classifiers and for different decision fusion methods. Conflicting classifier decisions are resolved by picking a decision $F_{1}$ at random, $F_{2}$ always from the classifier more accurate on the training set (here-speech), $F_{R}$ according to the higher reliability estimate, $F_{M}$ according to a higher margin-derived

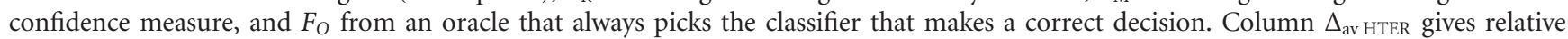
performances with respect to the oracle.

\begin{tabular}{l|rrr|rrr|rrrr}
\hline & \multicolumn{3}{|c|}{$\mathrm{g} 1$} & \multicolumn{3}{c|}{$\mathrm{g} 2$} & \multicolumn{3}{c}{ Average(g1, g2) } \\
\hline & HTER & FAR & FRR & HTER & FAR & FRR & HTER & FAR & FRR & $\Delta_{\text {av HTER }}$ \\
\hline Speech & 9.7 & 17.5 & 1.9 & 8.2 & 3.8 & 12.5 & 8.9 & 10.7 & 7.2 & 21.0 \\
Face & 26.7 & 25.2 & 28.2 & 22.0 & 34.6 & 9.3 & 24.3 & 29.9 & 18.8 & 8.5 \\
$F_{1}$ & 17.4 & 19.7 & 15.1 & 15.0 & 18.8 & 11.2 & 16.2 & 19.2 & 13.1 & 12.7 \\
$F_{2}$ & 9.7 & 17.5 & 1.9 & 8.2 & 3.8 & 12.5 & 8.9 & 10.7 & 7.2 & 23.0 \\
$F_{R}$ & $\mathbf{8 . 9}$ & $\mathbf{1 5 . 0}$ & $\mathbf{2 . 9}$ & $\mathbf{7 . 8}$ & $\mathbf{8 . 5}$ & $\mathbf{7 . 1}$ & $\mathbf{8 . 4}$ & $\mathbf{1 1 . 8}$ & $\mathbf{5 . 0}$ & $\mathbf{2 4 . 6}$ \\
$F_{M}$ & 10.6 & 11.5 & 9.6 & 9.7 & 14.5 & 4.8 & 10.1 & 13.0 & 7.2 & 20.3 \\
$F_{O}$ & 2.0 & 3.4 & 0.6 & 2.1 & 2.6 & 1.6 & 2.1 & 3.0 & 1.1 & 100 \\
\hline
\end{tabular}

TABLE 5: Agreement statistics.

\begin{tabular}{l|lcc}
\hline & Face wins & Speech wins & Unanimous \\
\hline g1 & $48(8.8 \%)$ & $102(18.7 \%)$ & $396(72.5 \%)$ \\
g2 & $43(7.9 \%)$ & $83(15.7 \%)$ & $417(76.4 \%)$ \\
\hline
\end{tabular}

the same data. Since in the case of biometric fusion the two classifiers operate on presumably independent datasets (face images and speech), the oracle fusion results should be rather understood as a gauge of the fusion scheme used. The fusion results, reported in terms of HTER and class accuracies are collected in Table 4.

As described in Section 6, the final decision could be unanimous, or be made upon the comparison of the modality reliability information in the case of disagreement. Table 5 shows the statistics of the decisions for the $\mathrm{g} 1$ and $\mathrm{g} 2$ groups.

\subsection{Discussion}

The experiments presented above confirm that the reliability measures can be put into effective use in the fusion of unimodal biometric verification decisions. The reliability approach outperformed the fusion scheme that uses marginderived confidence estimates. Decision-level fusion with margin-derived confidence measures proved to be an unsuccessful attempt altogether since the accuracies expressed in terms of 1-HTER were lower than those of the accuracies yielded by the speech modality alone. This result should be attributed to the fact that margin estimates are very sensitive to the relative shift of the development and testing distributions. The reliability estimates proved to be more robust to this effect, due to the use of the quality measures in the estimation process. The average fusion accuracy is superior to any of the unimodal approaches, and the accuracies for the datasets g1 and g2 are higher than that of the speech modality alone. However, the proposed fusion scheme is still far from perfect since it only reduced the gap between the best unimodal results and the hypothetical oracle-fusion results. In order to further diminish this difference, more sophis- ticated signal quality measures should be investigated, and score-based fusion schemes ought to be employed. It must be noted here that the speech part of the BANCA database does not offer similar qualitative spectrum of signals as the face part, few samples are of really decreased quality. This fact has its reflection in the plots of reliability estimates shown in Figure 6. Since on average speech-based decisions were labeled as more reliable, the fusion algorithm rarely made use of less reliable face data (see Table 5), and consequently the fusion results sport a limited improvement over speech results alone. It can be expected that given classification results of comparable reliability the proposed scheme would show a more pronounced improvement in fusion accuracy.

\section{CONCLUSIONS}

In this paper, we have demonstrated a method of performing multimodal fusion using unimodal classifier data, signal quality measures, and reliability estimates. We have shown on the example of face and speech modalities that the proposed method can be effectively applied to multimodal biometric fusion. Thanks to the use of the auxiliary quality information in the graphical model we managed to achieve an improved robustness to degraded signal conditions. We evaluated our method on a standard biometric multimodal database (BANCA), and compared the results of the proposed method to state-of-the-art approach of computing classification confidence margins. The proposed method based on reliability measures proved to outperform the alternative approaches.

\section{ACKNOWLEDGMENT}

This work was partly supported by the Swiss National Centre of Competence in Research IM2.MPR.

\section{REFERENCES}

[1] J. Short, J. Kittler, and K. Messer, "A comparison of photometric normalisation algorithms for face verification," in Proceedings of the 6th IEEE International Conference on Automatic Face 
and Gesture Recognition (FGR '04), pp. 254-259, Seoul, South Korea, May 2004.

[2] C. Barras and J.-L. Gauvain, "Feature and score normalization for speaker verification of cellular data," in Proceedings of IEEE International Conference on Acoustics, Speech, and Signal Processing (ICASSP'03), vol. 2, pp. 49-52, Hong Kong, April 2003.

[3] A. Ross, A. K. Jain, and J.-Z. Qian, "Information fusion in biometrics," in Proceedings of the 3rd International Conference on Audio- and Video-Based Biometric Person Authentication (AVBPA '01), pp. 354-359, Halmstad, Sweden, June 2001.

[4] F. Roli, J. Kittler, G. Fumera, and D. Muntoni, "An experimental comparison of classifier fusion rules for multimodal personal identity verification systems," in Proceedings of the 3rd International Workshop Multiple Classifier Systems (MCS '02), pp. 325-336, Cagliari, Italy, June 2002.

[5] J. Bigun, J. Fierrez-Aguilar, J. Ortega-Garcia, and J. GonzalezRodriguez, "Multimodal biometric authentication using quality signals in mobile communications," in Proceedings of the 12th International Conference on Image Analysis and Processing (ICIAP '03), pp. 2-11, Mantova, Italy, September 2003.

[6] J. Richiardi, P. Prodanov, and A. Drygajlo, "A probabilistic measure of modality reliability in speaker verification," in Proceedings of IEEE International Conference on Acoustics, Speech, and Signal Processing (ICASSP '05), vol. 1, pp. 709-712, Philadelphia, Pa, USA, March 2005.

[7] K. Kryszczuk, J. Richiardi, P. Prodanov, and A. Drygajlo, "Error handling in multimodal biometric systems using reliability measures," in Proceedings of the 13th European Signal Processing Conference (EUSIPCO '05), Antalya, Turkey, September 2005.

[8] N. Poh and S. Bengio, "Improving fusion with margin-derived confidence in biometric authentication tasks," in Proceedings of the 5th International Conference on Audio- and Video-Based Biometric Person Authentication (AVBPA '05), pp. 474-483, Hilton Rye Town, NY, USA, July 2005.

[9] N. Brümmer and J. du Preez, "Application-independent evaluation of speaker detection," Computer Speech \& Language, vol. 20, no. 2-3, pp. 230-275, 2006.

[10] C. Fredouille, J.-F. Bonastre, and T. Merlin, "Similarity normalization method based on world model and a posteriori probability for speaker verification," in Proceedings of the 6th European Conference on Speech Communication and Technology (EUROSPEECH '99), pp. 983-986, Budapest, Hungary, September 1999.

[11] K. Murphy, Dynamic Bayesian networks: representation, inference and learning, Ph.D. thesis, Computer Science Division, University of California - Berkeley, Berkeley, Calif, USA, July 2002.

[12] J. Richiardi, P. Prodanov, and A. Drygajlo, "Speaker verification with confidence and reliability measures," in Proceedings of IEEE International Conference on Acoustics, Speech, and Signal Processing (ICASSP '06), vol. 1, pp. 641-644, Toulouse, France, May 2006.

[13] E. Bailly-Bailliére, S. Bengio, F. Bimbot, et al., "The BANCA database and evaluation protocol," in Proceedings of the 4th International Conference on Audio- and Video-Based Biometric Person Authentication (AVBPA '03), J. Kittler and M. Nixon, Eds., vol. 2688 of Lecture Notes in Computer Science, pp. 625638, Guildford, UK, June 2003.

[14] J.-F. Bonastre, F. Wils, and S. Meignier, "ALIZE, a free toolkit for speaker recognition," in Proceedings of IEEE International Conference on Acoustics, Speech, and Signal Processing
(ICASSP '05), vol. 1, pp. 737-740, Philadelphia, Pa, USA, March 2005.

[15] D. Reynolds, A Gaussian mixture modeling approach to textindependent speaker identification, Ph.D. thesis, Georgia Institute of Technology, Atlanta, Ga, USA, 1992.

[16] K. Kryszczuk and A. Drygajlo, "On face image quality measures," in Proceedings of the 2nd Workshop on Multimodal User Authentication, Toulouse, France, May 2006.

[17] K. Messer, J. Kittler, M. Sadeghi, et al., "Face authentication competition on the BANCA database," in Proceedings of the 1st International Conference on Biometric Authentication (ICBA '04), pp. 8-15, Hong Kong, July 2004.

[18] C. Sanderson and S. Bengio, "Robust features for frontal face authentication in difficult image conditions," in Proceedings of the 4th International Conference on Audio- and Video-Based Biometric Person Authentication (AVBPA '03), pp. 495-504, Guildford, UK, June 2003.

Krzysztof Kryszczuk is a Ph.D. candidate at the Signal Processing Institute, Swiss Federal Institute of Technology Lausanne (EPFL). Before joining EPFL he was a Research Engineer at the National University of Singapore. He obtained his M.S. degree in psychology (cognitive systems engineering) from the Rensselaer Polytechnic Institute in 2001, and the M.S. degree in electrical engineering from the Lublin Institute of

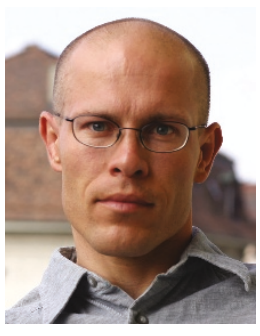
Technology in 1999. His research interests include statistical pattern recognition, image processing, biometrics, and human-machine interactions.

Jonas Richiardi received the B.Eng. (Hons) degree in electronic engineering with first class honours from the University of Essex, UK, in 2001. He received the M.Phil. degree in computer speech, text, and internet technology from the University of Cambridge, $\mathrm{UK}$, in 2002. He is currently pursuing the Ph.D. degree at Signal Processing Institute of the Swiss Federal Institute of Technology, Lausanne, Switzerland. He is a member of

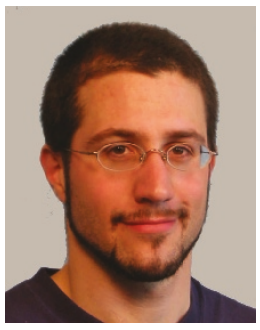
the IEEE and of the ISCA (International Speech Communication Association). His research interests include probabilistic modeling, classifier combination, graphical models, handwritten signature verification, and speech processing.

Plamen Prodanov was born in Varna Bulgaria, where he received his M.S. degree in telecommunications in 1998 at the Technical University of Varna, Bulgaria. After his graduation, he spent two years in the industry, working for radar development projects in the Signal Processing Laboratory at Cherno More Co. in Varna. Then he joined the Swiss Federal Institute of Technology, Lausanne (EPFL). From 2002 till

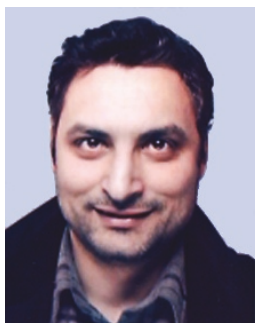
2006 he did a Ph.D. thesis titled "Error Handling in Multimodal Voice-Enabled Interfaces of Tour-Guide Robots Using Graphical Models" in the Speech Processing and Biometrics Group, EPFL. Since September 2006, he has joined the team of TBS Holding AG, where he is employed as a Research Engineer in the domain of 3D fingerprint recognition. 
Andrzej Drygajlo is the head of the Speech Processing and Biometrics Group at the Swiss Federal Institute of Technology at Lausanne (EPFL), where he conducts research on technological, methodological, and legal aspects of biometrics for security and forensic applications. In 1993 he created the EPFL Speech Processing Group (GTP) and then the EPFL Speech Processing and Biometrics Group (GTPB) and Bio-

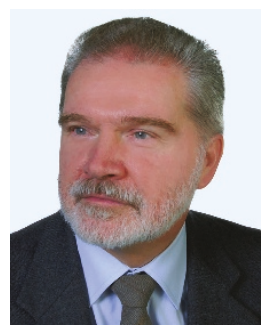
metrics Centre Lausanne. His research interests include biometrics, speech processing, and man-machine communication applications. He conducts research and teaches at the School of Engineering in EPFL and at the School of Criminal Sciences in the University of Lausanne. He participates in and coordinates numerous national and international projects and is member of various scientific committees. Among ongoing European research projects, the most relevant are the Network of Excellence "BioSecure" and COST 2101 Action "Biometrics for Identity Documents and Smart Cards." Recently, he has been elected as a Chairman of the COST 2101 Action. Dr. Drygajlo has been an advisor of numerous Ph.D. theses. He is the author/co-author of more than 100 research publications, including several book chapters, together with his own book. He is a member of the IEEE, EURASIP (European Association for Signal Processing) and ISCA (International Speech Communication Association) professional groups. 